\title{
Risk Factors for Dieulafoy Lesions in the Upper Gastrointestinal Tract
}

\author{
Hae Jin Shin, Jong Seok Ju, Ki Dae Kim, Seok Won Kim, Sung Hoon Kang, Sun Hyung Kang, \\ Hee Seok Moon, Jae Kyu Sung and Hyun Yong Jeong \\ Department of Gastroenterology, Chungnam National University Hospital, Chungnam National University School of Medicine, Daejeon, Korea
}

Background/Aims: The purpose of this study is to verify the risk factors associated with Dieulafoy lesion formation in the upper gastrointestinal tract.

Methods: A case-control study was performed by reviewing the electronic medical records of 42 patients who were admitted to a tertiary medical center in the Daejeon region for Dieulafoy lesions from September 2008 to October 2013, and the records of 132 patients who were admitted during the same period and who underwent endoscopic examination for reasons other than bleeding. We analyzed clinical and endoscopic findings retrospectively, and searched for risk factors associated with Dieulafoy lesion formation.

Results: All 42 patients diagnosed with Dieulafoy lesion had accompanying bleeding, and the location of the bleeding was proximal in 25 patients $(59.5 \%)$, the middle portion in seven patients (16.7\%), and distal in 10 patients $(23.8 \%)$. Antiplatelet agents $(p=0.022)$ and alcohol $(p=0.001)$ use showed statistically significant differences between the two groups. The odds ratios ( $95 \%$ confidence intervals) of the two factors were 2.802 (1.263 to 6.217) and 3.938 (1.629 to 9.521), respectively.

Conclusions: This study showed that antiplatelet agents and alcohol consumption were risk factors associated with Dieulafoy lesion formation in the upper gastrointestinal tract.

Key Words: Dieulafoy; Gastrointestinal bleeding; Endoscopic treatment; Antiplatelet agents; Alcohols

\section{INTRODUCTION}

Upper gastrointestinal bleeding is a medical emergency, and a Dieulafoy lesion is a potentially life-threatening cause of such bleeding. It constitutes $6 \%$ of upper gastrointestinal nonvariceal bleeding ${ }^{1,2}$ and $1 \%$ to $2 \%$ of all gastrointestinal bleeding. ${ }^{3,4}$ While the death rate due to Dieulafoy lesions is relatively low, it has a similar death rate to other causes of gastrointestinal bleeding. ${ }^{5}$

A Dieulafoy lesion is typically composed of closely attached submucosal arteries 1 to $3 \mathrm{~mm}$ in radius within various dis-

\footnotetext{
Received: July 14, 2014 Revised: October 13, 2014

Accepted: October 19, 2014

Correspondence: Sun Hyung Kang

Department of Gastroenterology, Chungnam National University Hospital, Chungnam National University School of Medicine, 282 Munhwa-ro, Junggu, Daejeon 301-721, Korea

Tel: +82-42-280-7207, Fax: +82-42-257-5753, E-mail: porrtos@cnuh.co.kr

(c) This is an Open Access article distributed under the terms of the Creative Commons Attribution Non-Commercial License (http://creativecommons.org/ licenses/by-nc/3.0) which permits unrestricted non-commercial use, distribution, and reproduction in any medium, provided the original work is properly cited.
}

tances from the gastric mucosa. Massive hemorrhage may result due to the erosion of the mucosa and arterial wall. ${ }^{6}$ Subintimal fibrosis and mucosal damage characteristically lack inflammatory reactions, and thus Dieulafoy lesions can be histologically differentiated from gastric ulcers.

The exact mechanism of bleeding in a Dieulafoy lesion has not yet been identified. Some authors have reported that this is due to the combined effects of atrophy of the gastric mucosa and ischemia. ${ }^{7}$ This means that pressure from vessels with a large diameter forms small erosions, which cause the rupture of the vessels into the lumen.

A Dieulafoy lesion clinically presents in the form of intermittent indolent gastrointestinal bleeding, ${ }^{8,9}$ most commonly in the stomach, ${ }^{3,5}$ and especially in the lesser curvature. ${ }^{5,10,11}$ Approximately $80 \%$ to $95 \%$ of Dieulafoy lesions are known to form within $6 \mathrm{~cm}$ of the gastroesophageal junction due to arterial vessels directly branching from the left gastric artery., ${ }^{72,13}$ One-third of Dieulafoy lesions form in places other than the stomach, mostly in the duodenum, ${ }^{14}$ followed by the colon. ${ }^{15,16}$ Elsewhere, Dieulafoy lesions have been reported in the esoph- 
agus, jejunum, ileum, ${ }^{12,17}$ rectum, ${ }^{18,19}$ and anal canal. ${ }^{20}$

The endoscopic diagnostic rate of Dieulafoy lesions is known to be over $90 \%, 4,5$ but diagnosis may be difficult during the initial bleeding stage due to the small size of the lesion and intermittent bleeding patterns. Because of this, $6 \%$ of patients require more than three endoscopic examinations for precise diagnosis. $^{5}$

The traditional treatment method for Dieulafoy lesions has been surgery. However, due to technological advancements in endoscopic treatment, the need for surgery has largely decreased, and the death rate has also dropped from $80 \%$ to $8.6 \% .{ }^{21}$ Currently, the most important treatment method for Dieulafoy lesions is endoscopy, which is safe and highly effective for early hemostasis. ${ }^{1,22}$ The success rate of endoscopic treatment ranges from $70 \%$ to $98 \%,{ }^{23-26}$ and the incidence of reoccurrence and complications is low. ${ }^{22,24,27-31}$ Many methods of endoscopic treatment are used, such as the injection of sclerosing agents, high temperature coagulation, and apparatus such as band clipping or hemoclipping. The actual method of endoscopic treatment depends on the experience and decision of the endoscopists.

A Dieulafoy lesion is an important cause of bleeding without a clear etiology. It is important to know the risk factors associated with Dieulafoy lesion formation, but these are currently not well understood. The objective of this study is to verify the associated risk factors of Dieulafoy lesion formation in the upper gastrointestinal tract, and to assess the effect of the endoscopic treatment of Dieulafoy lesions with bleeding of the upper gastrointestinal tract.

\section{MATERIALS AND METHODS}

\section{Patients}

A patient-control study was performed by reviewing the electronic medical records of 42 patients who were diagnosed with Dieulafoy lesions after receiving emergency endoscopic treatment due to hematemesis, melena, or hematochezia. These patients were admitted to a tertiary medical facility in the Daejon region from September 2008 to October 2013. A group of 132 patients who received endoscopic treatment for reasons other than bleeding, and who were admitted during the same period, served as the control group.

The 42 patients who were diagnosed with Dieulafoy lesions received basic treatment before receiving emergency endoscopic treatment. All procedures were performed within at least 6 hours of admission to the emergency room by three endoscopic specialists. Consent forms were signed by all patients or their families. All patients received intravenous proton pump inhibitors before emergency endoscopic treatment, and some received intravenous analgesics if necessary. However, no pa- tients received intravenous sedatives. Follow-up endoscopy was performed within 3 days of the initial endoscopic treatment, and in patients with rebleeding, emergency endoscopic treatment was performed on the day of bleeding. Follow-up was performed within 3 days. The 132 patients in the control group were selected from patients who were admitted during the same period who received endoscopic treatment for reasons other than bleeding. Patient information was collected by reviewing electronic medical records, which included information regarding basic biographical data, medication history (nonsteroidal anti-inflammatory drugs [NSAIDs], anticoagulants, or antiplatelet agents), initial hemodynamic conditions, blood test results, and methods of endoscopic treatment and their results (rebleeding, survival and death, or need for surgery). For the precise selection of the Dieulafoy lesion patient group, electronic medical records were acquired for patients diagnosed with Dieulafoy lesions, and patients who received a definite diagnosis from endoscopic specialists who reexamined their endoscopic records were included in the study.

\section{Definitions}

Endoscopic diagnoses of Dieulafoy lesions were made according to the following three criteria: ${ }^{32}(1)$ active arterial spurting or micropulsatile streaming from minute $(<3 \mathrm{~mm})$ mucosal defects; (2) visualization of a protruding vessel with or without active bleeding within a minute mucosal defect with normal surrounding mucosa; or (3) a densely adherent clot with a narrow attachment point to a minute mucosal defect or normal-appearing mucosa.

Initial failure of endoscopic hemostasis was defined as continued active bleeding despite endoscopic treatment or hematemesis, melena, hematochezia, and hemodynamically unstable conditions (systolic blood pressure [SBP] below 100 $\mathrm{mm} \mathrm{Hg}$, heart rate exceeding 100 per minute, decrease of standing SBP greater than $20 \mathrm{~mm} \mathrm{Hg}$, and heart rate 20 per min) within 12 hours of initial endoscopic hemostasis. Rebleeding was suspected with hematemesis, melena and hematochezia, or hemodynamically unstable conditions, or with decreased hemoglobin exceeding $2 \mathrm{~g} / \mathrm{dL}$ within 24 hours and was diagnosed if bleeding was endoscopically confirmed the location of the previously treated lesion.

Potential factors included the anticoagulants warfarin, rivaroxaban, and dabigatran, the antiplatelet agents aspirin, clopidogrel, and cilostazol, and the NSAIDs tramadol, aceclofenac, dexibuprofen, meloxicam, ketorolac, naproxen, celecoxib, nimesulide, sulindac, and zaltoprofen.

\section{Statistical analysis}

In order to verify the statistical meaning of baseline characteristics of Dieulafoy lesion formation between the two groups, 
cross analyses using the chi-square test, Student $t$-test, and logistic regression analysis were performed. In addition, frequency analysis was performed in order to verify the associations of diseases between the two groups. A $p<0.05$ indicated statistical significance, and all statistical analysis was performed using SPSS version 21.0 (IBM Co., Armonk, NY, USA).

\section{RESULTS}

Of all the patients who underwent endoscopic treatment during the study period, 51 patients were diagnosed with Dieulafoy lesions. However, upon further review of their endoscopic records by endoscopic specialists, nine were excluded, and 42 patients were definitively diagnosed with Dieulafoy lesions and ultimately included in the study (Table 1). All 42 patients had accompanying bleeding, and the location of the bleeding was proximal (cardia to upper body) in 25 patients (59.5\%), in the middle (mid-body) portion in seven patients (16.7\%), and distal (lower body to pylorus) in 10 patients (23.8\%). The initial endoscopic treatment method was hypertonic saline-epinephrine injection in one patient (2.4\%), hemoclipping in 19 patients (45.2\%), and a combination of hypertonic saline-epinephrine injection and hemoclipping in 22 patients (52.4\%). All 42 patients who received initial endoscopic treatment achieved primary hemostasis, while no patient failed to achieve successful endoscopic hemostasis. Rebleeding occurred in four patients (9.5\%) within 1 to 3 days of initial endoscopic treatment, but all achieved permanent hemostasis with additional endoscopic hemostatic treatment (hemoclipping in one patient and a combination of hypertonic saline-epinephrine injection and hemoclipping in three patients) (Fig. 1).

The correlation of Dieulafoy lesion formation and sex, the administration of NSAIDs, anticoagulants, antiplatelet agents, associated diseases (diabetes, hypertension, etc.), and smoking and alcohol consumption between the patient and control groups were analyzed (Table 2 ).

Upon analysis, antiplatelet agents $(p=0.022)$ and alcohol ( $p=0.001)$ showed statistically significant differences between

Table 1. Location of the Dieulafoy Lesion and Hemostatic Methods $(n=42)$

\begin{tabular}{lc}
\hline \multicolumn{1}{c}{ Parameter } & No. (\%) \\
\hline Location & $25(59.5)$ \\
Proximal stomach & $7(16.7)$ \\
Mid-stomach & $10(23.8)$ \\
Distal stomach & \\
Hemostatic methods & $1(2.4)$ \\
HSE injection & $19(45.2)$ \\
Hemoclipping & $22(52.4)$ \\
HSE injection+hemoclipping &
\end{tabular}

HSE, hypertonic saline-epinephrine.

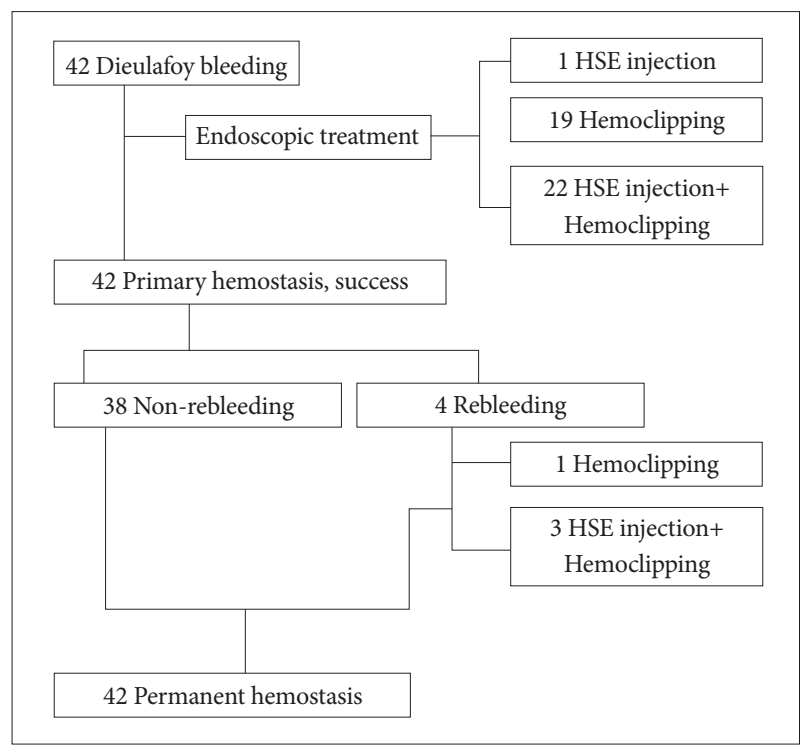

Fig. 1. Summary of the management pathways and treatment outcomes. HSE, hypertonic saline-epinephrine.

Table 2. Baseline Characteristics of the Dieulafoy Lesion and Control Group

\begin{tabular}{|c|c|c|c|}
\hline Parameter & Dieulafoy lesion group $(n=42)$ & Control group $(n=132)$ & $p$-value \\
\hline Age, yr & $64.8 \pm 16.2$ & $61.9 \pm 16.5$ & 0.326 \\
\hline Sex, male:female & $14: 28(33.3: 66.7)$ & $66: 66(50: 50)$ & 0.059 \\
\hline NSAIDs intake & $7(16.7)$ & $26(19.7)$ & 0.663 \\
\hline Anticoagulant intake & $3(7.1)$ & $4(3.0)$ & 0.237 \\
\hline Antiplatelet intake & $19(45.2)$ & $35(26.5)$ & 0.022 \\
\hline Smoking & $13(31.0)$ & $25(18.9)$ & 0.101 \\
\hline Alcohol & $16(38.1)$ & $20(15.2)$ & 0.001 \\
\hline Diabetes & $15(35.7)$ & $45(34.1)$ & 0.847 \\
\hline Hypertension & $20(47.6)$ & $60(45.5)$ & 0.806 \\
\hline Concomitant disease (excluding diabetes, hypertension) & $32(76.2)$ & $117(88.6)$ & 0.055 \\
\hline
\end{tabular}

Values are presented as mean \pm SD or number (\%).

NSAID, nonsteroidal anti-inflammatory drug. 
the two groups. A logistic regression model showing the contribution rate of these two factors showed that the odds ratios (95\% confidence interval) were 2.802 (1.263 to 6.217) for antiplatelet agents and 3.938 (1.629 to 9.521) for alcohol (Table 3).

In the analysis of the method of endoscopic treatment that resulted in rebleeding in four patients, none was due to hypertonic saline-epinephrine injection, one was due to hemoclipping, and three were due to a combination of hypertonic saline-epinephrine injection and hemoclipping. However, as the sample size was small, there was no statistically significant difference $(p=0.626)$ (Table 4$)$.

The methods and results of endoscopic treatment of the patients are summarized in detail in Fig. 1.

The average outpatient follow-up period for the 42 patients diagnosed with Dieulafoy lesions was 12 months (range, 5 to 24). No patient showed rebleeding during the follow-up period, and there were no complications associated with the treatment.

\section{DISCUSSION}

The Dieulafoy lesion was first described in 1884 by Gallarden, ${ }^{7,11,15}$ and in 1898, the French surgeon George Dieulafoy found common characteristics, which he named the Dieulafoy lesions, in three patients.

A Dieulafoy lesion is an important cause of life-threatening upper gastrointestinal bleeding. It comprises $6 \%$ of all upper gastrointestinal bleeding ${ }^{1,2}$ and $1 \%$ to $2 \%$ of all gastrointestinal bleeding. ${ }^{3,4}$

In this study, the clinical features of patients with Dieulafoy lesions were similar to the results of previous studies. ${ }^{10,11,22,29,33}$ During the study period, Dieulafoy lesions occurred in women two times as often as in men, and the patients' average age

Table 3. Logistic Regression Model Analysis of the Two Factors

\begin{tabular}{lcc}
\hline \multicolumn{1}{c}{ Parameter } & Odds ratio & 95\% Confidence interval \\
\hline Antiplatelet intake & 2.802 & $1.263-6.217$ \\
Alcohol & 3.938 & $1.629-9.521$ \\
\hline
\end{tabular}

was 64.8 years. Analysis showed that there were statistically significant differences between the study group and the control group with respect to antiplatelet agents $(p=0.022)$ and drinking $(p=0.001)$.

Some previous studies have suggested a correlation between Dieulafoy lesions and the administration of NSAIDs or aspirin, and there have been reports of NSAIDs and aspirin causing erosive gastritis through necrosis of vessel walls, leading to the rupture of submucosal vessels. ${ }^{711}$ In this study, the rates of administration of NSAIDs, anticoagulants, and antiplatelet agents were $16.7 \%, 7.1 \%$, and $45.2 \%$, respectively. However, only antiplatelet agents were significantly associated with Dieulafoy lesions.

A previous study showed the effects of chronic drinking on the gastric mucosa of animals, ${ }^{34}$ and the results are were follows. Chronic drinking firstly resulted in the damage of the mucosal epithelium, secondly in the decrease of mucosal cells near the pylorus, and thirdly in the decrease of gastric mucosal thickness. It is probable that chronic drinking damages gastric mucosa through such mechanisms, which as a result increases the risk of Dieulafoy lesion formation.

In this study, over $80 \%$ of patients with Dieulafoy lesions had associated diseases. Some authors have reported that associated diseases such as cardiac disorders, hypertension, and renal failure alter the normal process of angiogenesis, resulting in the formation of abnormal vessels of a certain diameter, which increases the prevalence rate of Dieulafoy lesions. ${ }^{35}$

Endoscopy is a key treatment method for Dieulafoy lesions. It is very safe and effective, leading to successful initial hemostasis. ${ }^{1,22}$ Many methods of endoscopic treatment leading to successful initial hemostasis have been reported, including injection of sclerosing agents, ${ }^{22,23,36}$ high temperature coagulation, ${ }^{23,33}$ and the use of apparatus such as band clipping ${ }^{37}$ or hemoclipping. ${ }^{38}$ The success rate of hemostasis for the various endoscopic treatment methods ranges from $70 \%$ to $98 \%{ }^{23-26}$ Recent studies have shown that band clipping or hemoclipping increases the success rate of primary hemostasis while decreasing the need for additional endoscopic treatment. ${ }^{37}$ Theoretically, as the use of apparatus results in less damage to sur-

Table 4. An Analysis of the Correlation between the Hemostatic Methods and Rebleeding

\begin{tabular}{|c|c|c|c|c|}
\hline \multirow{2}{*}{ Parameter } & \multicolumn{2}{|c|}{ Parameter } & \multirow{2}{*}{ Total } & \multirow{2}{*}{$p$-value } \\
\hline & No & Yes & & \\
\hline Hemostatic methods & & & & 0.626 \\
\hline HSE injection & $1(100)$ & 0 & $1(100)$ & \\
\hline Hemoclipping & $18(94.7)$ & $1(5.3)$ & $19(100)$ & \\
\hline HSE injection+hemoclipping & $19(86.4)$ & $3(13.6)$ & $22(100)$ & \\
\hline Total & $38(90.5)$ & $4(9.5)$ & & \\
\hline
\end{tabular}

Values are presented as number (\%).

HSE, hypertonic saline-epinephrine. 
rounding tissue compared to the injection of sclerosing agents or high temperature coagulation methods, it is currently being proposed as the primary treatment method for Dieulafoy lesions. ${ }^{31,39}$ In addition, another study has reported that the combination of hemoclipping and aethoxysklerol is the most effective treatment. ${ }^{40}$

In this study, all 42 patients (100\%) diagnosed with Dieulafoy lesions successfully achieved primary hemostasis, which is a higher success rate than reported in previous studies. ${ }^{21,39,41}$ Four patients $(9.5 \%)$ had rebleeding within 1 to 3 days of initial endoscopic treatment, but all achieved permanent hemostasis with additional endoscopic hemostatic treatment (hemoclipping in one patient and a combination of hypertonic saline-epinephrine injection and hemoclipping in three patients). Analysis of the association between rebleeding and the method of endoscopic treatment in the four patients showed that a combination of hypertonic saline-epinephrine injection and hemoclipping resulted in rebleeding in three patients. However, this was not statistically significant $(p=0.626)$. Anal$y$ sis of the initial hemoglobin values and hemodynamic conditions, and the amount of total red blood cell transfusion during the admission period of the four patients with rebleeding showed that the selection of combination treatment rather than a single treatment method was probably due to the severity of the bleeding.

The long-term prognosis of the Dieulafoy lesion patients who received endoscopic treatment was very good. ${ }^{27,37,42} \mathrm{Im}-$ mediate diagnosis and treatment generally results in a very low death rate. In this study, of the 42 patients diagnosed with Dieulafoy lesions, 16 patients (38\%) showed hemodynamically unstable conditions, while all 42 patients (100\%) showed accompanying active bleeding. All 42 patients survived the 5 -year study period, while two patients died during the followup period due to chronic renal failure and multiple myeloma, both of which were unrelated to the Dieulafoy lesion. The average outpatient follow-up period was 12 months (range, 5 to 24); no patients showed rebleeding during that time, and there were no complications associated with the treatment.

This study has a few limitations. First, due to the low prevalence rate of Dieulafoy lesions, the sample size is small. This is also a retrospective study, and as the electronic medical records were not created with research objectives in mind, there is a possibility that information regarding medication history and smoking and drinking may not be accurate. Additionally, there are limitations in giving statistical significance to the results as the sample size is small. Second, there is a possibility that the composition of the control group may be different from that of the normal population. Although the control group was selected during the study period from among the admitted patients in order to reproduce the composition of the normal population, there are admittedly limitations in doing this properly. Third, as all patients diagnosed with Dieulafoy lesions that were registered for this study were diagnosed and treated at only this hospital, there are limitations in the overall representativeness of the results.

Despite these limitations, this study shows that the risk factors associated with Dieulafoy lesion formation in the upper gastrointestinal tract are antiplatelet agents and drinking.

\section{Conflicts of Interest}

The authors have no financial conflicts of interest.

\section{REFERENCES}

1. Stark ME, Gostout CJ, Balm RK. Clinical features and endoscopic management of Dieulafoy's disease. Gastrointest Endosc 1992;38:545550.

2. Strong RW. Dieulafoy's disease: a distinct clinical entity. Aust N Z J Surg 1984;54:337-339.

3. Chaer RA, Helton WS. Dieulafoy's disease. J Am Coll Surg 2003;196: 290-296.

4. Marangoni G, Cresswell AB, Faraj W, Shaikh H, Bowles MJ. An uncommon cause of life-threatening gastrointestinal bleeding: 2 synchronous Dieulafoy lesions. J Pediatr Surg 2009;44:441-443.

5. Lara LF, Sreenarasimhaiah J, Tang SJ, Afonso BB, Rockey DC. Dieulafoy lesions of the GI tract: localization and therapeutic outcomes. Dig Dis Sci 2010;55:3436-3441.

6. Veldhuyzen van Zanten SJ, Bartelsman JF, Schipper ME, Tytgat GN. Recurrent massive haematemesis from Dieulafoy vascular malformations: a review of 101 cases. Gut 1986;27:213-222.

7. Lee YT, Walmsley RS, Leong RW, Sung JJ. Dieulafoy's lesion. Gastrointest Endosc 2003;58:236-243.

8. Kasapidis P, Georgopoulos P, Delis V, Balatsos V, Konstantinidis A, Skandalis N. Endoscopic management and long-term follow-up of Dieulafoy's lesions in the upper GI tract. Gastrointest Endosc 2002;55: 527-531.

9. Parra-Blanco A, Takahashi H, Méndez Jerez PV, et al. Endoscopic management of Dieulafoy lesions of the stomach: a case study of 26 patients. Endoscopy 1997;29:834-839.

10. Schmulewitz N, Baillie J. Dieulafoy lesions: a review of 6 years of experience at a tertiary referral center. Am J Gastroenterol 2001;96:16881694.

11. Chung IK, Kim EJ, Lee MS, et al. Bleeding Dieulafoy's lesions and the choice of endoscopic method: comparing the hemostatic efficacy of mechanical and injection methods. Gastrointest Endosc 2000;52:721-724.

12. al-Mishlab T, Amin AM, Ellul JP. Dieulafoy's lesion: an obscure cause of GI bleeding. J R Coll Surg Edinb 1999;44:222-225.

13. Anireddy D, Timberlake G, Seibert D. Dieulafoy's lesion of the esophagus. Gastrointest Endosc 1993;39:604.

14. Pollack R, Lipsky H, Goldberg RI. Duodenal Dieulafoy’s lesion. Gastrointest Endosc 1993;39:820-822.

15. Alshumrani G, Almuaikeel M. Angiographic findings and endovascular embolization in Dieulafoy disease: a case report and literature review. Diagn Interv Radiol 2006;12:151-154.

16. Katsinelos P, Pilpilidis I, Paroutoglou G, et al. Dieulafoy-like lesion of the colon presenting with massive lower gastrointestinal bleeding. Surg Endosc 2004;18:346.

17. Dulic-Lakovic E, Dulic M, Hubner D, et al. Bleeding Dieulafoy lesions of the small bowel: a systematic study on the epidemiology and efficacy of enteroscopic treatment. Gastrointest Endosc 2011;74:573-580.

18. Yoshikumi Y, Mashima H, Suzuki J, et al. A case of rectal Dieulafoy's ulcer and successful endoscopic band ligation. Can J Gastroenterol 2006; 
20:287-290.

19. Nunoo-Mensah JW, Alkari B, Murphy GJ, Watson AJ. Rectal Dieulafoy lesions. J Am Coll Surg 2008;206:388-389.

20. Firat O, Karaköse Y, Calişkan C, Makay O, Ozütemiz O, Korkut MA. Dieulafoy's lesion of the anal canal: report of a case. Turk J Gastroenterol 2007;18:265-267.

21. Baxter M, Aly EH. Dieulafoy's lesion: current trends in diagnosis and management. Ann R Coll Surg Engl 2010;92:548-554.

22. Baettig B, Haecki W, Lammer F, Jost R. Dieulafoy's disease: endoscopic treatment and follow up. Gut 1993;34:1418-1421.

23. Skok P. Endoscopic hemostasis in exulceratio simplex-Dieulafoy's disease hemorrhage: a review of 25 cases. Endoscopy 1998;30:590-594.

24. Pointner R, Schwab G, Königsrainer A, Dietze O. Endoscopic treatment of Dieulafoy's disease. Gastroenterology 1988;94:563-566.

25. Lin HJ, Lee FY, Tsai YT, Lee SD, Lee CH, Kang WM. Therapeutic endoscopy for Dieulafoy's disease. J Clin Gastroenterol 1989;11:507-510.

26. Asaki S, Sato H, Nishimura T, et al. Endoscopic diagnosis and treatment of Dieulafoy's ulcer. Tohoku J Exp Med 1988;154:135-141.

27. Sone Y, Kumada T, Toyoda H, Hisanaga Y, Kiriyama S, Tanikawa M. Endoscopic management and follow up of Dieulafoy lesion in the upper gastrointestinal tract. Endoscopy 2005;37:449-453.

28. Romãozinho JM, Pontes JM, Lérias C, Ferreira M, Freitas D. Dieulafoy’s lesion: management and long-term outcome. Endoscopy 2004;36:416420.

29. Norton ID, Petersen BT, Sorbi D, Balm RK, Alexander GL, Gostout CJ. Management and long-term prognosis of Dieulafoy lesion. Gastrointest Endosc 1999;50:762-767.

30. Yamaguchi Y, Yamato T, Katsumi N, et al. Short-term and long-term benefits of endoscopic hemoclip application for Dieulafoy's lesion in the upper GI tract. Gastrointest Endosc 2003;57:653-656.

31. Nikolaidis N, Zezos P, Giouleme O, et al. Endoscopic band ligation of Dieulafoy-like lesions in the upper gastrointestinal tract. Endoscopy 2001;
33:754-760.

32. Dy NM, Gostout CJ, Balm RK. Bleeding from the endoscopically-identified Dieulafoy lesion of the proximal small intestine and colon. Am J Gastroenterol 1995;90:108-111.

33. Iacopini F, Petruzziello L, Marchese M, et al. Hemostasis of Dieulafoy's lesions by argon plasma coagulation (with video). Gastrointest Endosc 2007;66:20-26.

34. Lee EA, Kim JB, Kim BS, et al. Morphological effect of chronic alcohol drinking upon the gastric mucosa of rats. Korean J Anat 2000;33:519-527.

35. Ding YJ, Zhao L, Liu J, Luo HS. Clinical and endoscopic analysis of gastric Dieulafoy's lesion. World J Gastroenterol 2010;16:631-635.

36. Laine L. Multipolar electrocoagulation in the treatment of active upper gastrointestinal tract hemorrhage. A prospective controlled trial. N Engl J Med 1987;316:1613-1617.

37. Park CH, Joo YE, Kim HS, Choi SK, Rew JS, Kim SJ. A prospective, randomized trial of endoscopic band ligation versus endoscopic hemoclip placement for bleeding gastric Dieulafoy's lesions. Endoscopy 2004;36: 677-681.

38. Park CH, Sohn YH, Lee WS, et al. The usefulness of endoscopic hemoclipping for bleeding Dieulafoy lesions. Endoscopy 2003;35:388-392.

39. Alis H, Oner OZ, Kalayci MU, et al. Is endoscopic band ligation superior to injection therapy for Dieulafoy lesion? Surg Endosc 2009;23:1465-1469.

40. Cui J, Huang LY, Liu YX, et al. Efficacy of endoscopic therapy for gastrointestinal bleeding from Dieulafoy's lesion. World J Gastroenterol 2011;17: 1368-1372.

41. Cheng CL, Liu NJ, Lee CS, et al. Endoscopic management of Dieulafoy lesions in acute nonvariceal upper gastrointestinal bleeding. Dig Dis Sci 2004;49:1139-1144.

42. Yilmaz M, Ozütemiz O, Karasu Z, et al. Endoscopic injection therapy of bleeding Dieulafoy lesion of the stomach. Hepatogastroenterology 2005;52:1622-1625. 